\title{
CBR Transportation of VBR MPEG-2 Video Traffic for Video-On-Demand in ATM Networks
}

\author{
Jian Ni , Tao Yang \\ Dept. of Industrial Eng. \\ Technical University of Nova Scotia \\ Halifax, Nova Scotia, B3J 2X4 \\ e-mail: yangt@tuns.ca
}

\author{
Danny H K Tsang \\ Dept. of Electrical \& Electronic Eng. \\ Hong Kong Univ. of Science \& Technology \\ Kowloon, Hong Kong
}

\begin{abstract}
In this paper, a CBR transport scheme for VBR MPEG-2 video-on-demand traffic in ATM networks is proposed. The basic idea is to smooth the VBR traffic to match a given bandwidth. In order to keep the continuous playback of the video, our scheme requires a suitable amount of buffer space at the receiver side and introduces an initial playback delay. We study these two performance measures as functions of allocated bandwidth. For given buffer size and bandwidth, it is sometimes necessary to control the transmission in order to satisfy the required QoS. Two control methods are examined and the corresponding minimum buffer requirements for the given QoS and bandwidth are investigated.
\end{abstract}

\section{Introduction}

As a potential major traffic in future ATM-based B-ISDN, video has generated a lot of research activities. Among them, a large proportion were on the statistical characteristics of the video sequences $[4,7,9,11,12]$. From these studies, it is clear that VBR MPEG-2 traffic is extremely content-dependent and thus almost unpredictable. In comparison with other VBR traffic (e.g. video conferencing and on-off sources) such a traffic pattern is far more complicated. Hence, an exact bandwidth estimation for such traffic will be very complicated and require a considerable amount of computation time which would not be acceptable for real-time implementations.

Since most of the difficulties in handling VBR traffic are caused by its unpredictable instant rate, it was therefore suggested to do some kind of smoothing on the traffic before feeding it into the network. The cost for traffic smoothing is the extra processing delay. According to the length of the smoothing period, smoothing can be a long-term smoothing or a short-term smoothing. Short-term smoothing schemes can only reduce the rate variation to some extent but still produce a VBR traffic sequence. Such a scheme, called the macro-frame scheme, has been studied in $[6,10]$ and it was found that it can really save some bandwidth. Long-term smoothing scheme has the potential ability to produce a real CBR traffic. The problems are how to balance the advantages and disadvantages introduced.

In this paper, we propose a CBR transportation technique for VBR MPEG-2 video-on-demand (a long-term smoothing scheme). Before describing the details of the method, it is necessary to point out that 1) our CBR transportation method is not the one that is achieved in the MPEG-2 encoder by dynamically adjusting the quantization scale to match the target constant bit rate output and 2) our method is also applicable to other non-real time VBR traffic although it is proposed for MPEG-2 traffic here. As will be shown in the paper, our CBR transportation method can save a significant amount of bandwidth at the cost of only some acceptable initial playback delay and decoder buffer. Even in cases where buffer size is limited, the scheme can still be applied using rate control.

The organization of this paper is as follows. Statistical characteristics of VBR MPEG-2 sequences are summarized in the next section. In section 3, the proposed CBR transportation scheme is discussed. Two controlled CBR transportation schemes are discussed in section 4. Finally, section 5 concludes the paper.

\section{Characteristics of MPEG-2 Sequence}

MPEG-2 coding algorithm carries out the source compression from both temporal and spatial directions. Generally, an MPEG-2 encoder can generate three types of frames, namely, the intra-frames ( $I$ frames), the predicted frames ( $P$ frames) and the bi-directional frames ( $B$ frames). An $I$ frame is the start of a certain coding period and it is obtained by simply encoding an individual picture without utilizing any related information in the past frames. While a $P$ frame contains the information that is predicted from the most previous $I$ or $P$ frames. A $B$ frame is generated by considering the contents in both the past and the future $I$ or $P$ frames immediately adjacent to it. Therefore, the coded video sequence is in the format like $I B B P B B P B B P I B B P \ldots$

The traffic sequence used throughout this paper are composed of 66 pieces of short movie samples with various scene contents, including both scenes with quick background change and scenes without background change. The total length of the 66 sub-sequences is around 19,000 frames and this will last for about 633.33 seconds. In order to have a sufficient long sequence for investigation, we randomly mixed the 66 subsequences to compose several long sequences of around 59,467 frames.

Our statistical analysis of the composed sequence and the sequences of different types of frames showed results similar to what were reported in $[4,11]$. First, the sequences present a pseudo-periodic property. The reason behind this is the periodic I $B B P B B P B B I B B P \ldots$ frame structure. Second, $I$ frames generally have relatively higher rates while $P$ and $B$ frames are of relatively lower rates. The large variation and the pseudo-periodic property of MPEG sequences is caused by the alternate appearing of the three types of frames. Third, the distribution histograms of the sequences showed that it is difficult to find a suitable distribution model to fit it due to the 
double-peak shape. Fourth, the autocorrelation function of MPEG-2 sequences also present some kind of periodic property and it is no longer the monotonous decreasing function of the length of the leg.

The complicated statistical characteristics of MPEG-2 traffic make it very difficult to do the bandwidth allocation. First of all, it is difficult, if not impossible, to model such a traffic as those done for on-off VBR traffic or VBR video conferencing traffic and consequently the effective bandwidth method [1,2, $3,8]$ is no longer applicable. Secondly, since the traffic rate distribution is far more complicated than a normal distribution, the stationary Gaussian approximation $[1,5]$ will also be far from accurate. In the following section, we will try to solve this problem in a different way. The method is to transport VBR MPEG-2 video traffic in a CBR manner rather than estimate the required bandwidth based on the source statistics.

\section{CBR Transportation Scheme}

The proposed CBR transportation scheme for VBR MPEG-2 video-on-demand traffic works as follows. The video sequence is transported at the speed $C$ (cells per frame interval). If the cell rate of a frame is larger than $C$, the extra cells will be transported in succeeding frame intervals. If the cell rate of a frame is less than $C$, the remaining bandwidth will be used to transmit the cells in succeeding frames. Clearly, the scheme is equivalent to a long-term smoothing process with smoothing period being the whole video sequence.

In order to ensure continuous playback of the video, it is required that all the cells in a frame must be able to reach the receiver at the time when the frame is decoded. This requirement is not difficult to satisfy if the cell rate of the frame is less than the given bandwidth. If the cell rate of a frame is large than the given bandwidth, some cells in the frame has to be delayed for a while, and if a number of high cell rate frames arrive successively, the delay will be accumulated gradually. To avoid the discontinuous playback that might be caused by such a delay, a certain amount of initial playback delay is necessary (i.e. to start the playback after the required initial delay). The minimum initial delay is determined by the maximum number of accumulated cells that are behind their scheduled frame departure time. Meanwhile, in order to store the cells arriving during the initial delay period and arriving prior to their scheduled frame decoding time, a certain amount of receiver buffer is also required. It is clear that the receiver buffer size is determined by the larger value between the number of cells arriving in the initial playback delay period and the maximum number of accumulated cells arriving prior to their scheduled frame decoding time. What we are interested here are the relationships between the given bandwidth and the above issues.

The initial playback delay can be calculated as follows. Suppose the video sequence is represented by $\left\{X_{i}, i=0,1, \ldots, N\right\}$, where $X_{i}$ is the number of cells in frame $i$ and $N+1$ is the total number of frames in the sequence. Then for the given bandwidth $C$, after the $(n+1)$ th frame interval, the number of cells that should be but have not yet been transported is
Suppose

$$
Y_{n}=\sum_{i=0}^{n}\left(X_{i}-C\right)
$$

$$
B_{t}=\max \left\{0, Y_{n} ; n=0,1, \ldots, N\right\} .
$$

The maximum initial playback delay $D$ is determined by $B_{t}$ as

$$
D=B_{t} / C
$$

since $B_{t}$ is the maximum number of cells to be transported. The decoder buffer size can be calculated as follows. After the $(n+1)$ th frame interval, the number of cells that have been transported before their scheduled playback time is $\sum_{i=0}^{n}\left(C-X_{i}\right)$, i.e., $-Y_{n}$. Then, the receiver buffer required to store these cells is of the size of

$$
B_{r 1}=-\min \left\{0, Y_{n} ; n=0,1, \ldots, N\right\} .
$$

Besides this, the buffer should also be able to store those cells arriving in the initial delay period and the minimum buffer size required will be $B_{r 2}=B_{t}$. Thus, the total receiver buffer required will be

$$
B_{r}=\max \left\{B_{r 1}, B_{r 2}\right\} .
$$

Theoretical solutions to the above equations require an accurate model for the video sequence. To model an original VBR MPEG-2 video sequence is extremely difficult and no suitable modelling method is available yet. In $[6,10]$, a method to model a smoothed VBR MPEG-2 sequence was proposed. In this modelling method, a group of frames between two adjacent $I$ frames (typically 12 frames) in the sequence is defined as a macro-frame (or $M$-frame) and the cell rate variation in an $M$-frame is smoothed over the whole $M$ frame interval. Hence, there are only cell rate variations among different $M$-frames. Since what will be calculated in the above equations are only related to the accumulated difference between the given constant bandwidth and the instant variable cell rate, there is then no difference to use either a frame based or an $M$-frame based calculation.

The model proposed in [10] for $M$-frame smoothed VBR MPEG-2 video sequence is a discrete time Markov modulated deterministic process (D-MMDP) [13] which can be generated by the superposition of a certain number of identical, independent two-state mini-sources. There are totally five basic parameters, the cell generating rates of the mini-source at the two states respectively, the two transition probabilities between the two states and the number of mini-sources, to be determined for the model and they can be obtained by matching the five statistical characteristics (peak rate, bottom rate, mean rate, standard deviation and autocorrelation function coefficient) of the real sequence and the model generated sequence correspondingly. Results obtained in [10] shows that the model is sufficiently accurate. Hence, in the following analysis, we will use such a D-MMDP model.

Suppose the D-MMDP used to model an M-frame smoothed VBR MPEG-2 video sequence is composed of $M$ minisources, each having two states with cell generating rates being respectively $R_{l}$ and $R_{h}$. The transition probability from $R_{l}$ to $R_{h}$ is $\alpha$ and from $R_{h}$ to $R_{l}$ is $\beta$. If we represent the 
model generated $M$-frame video sequence as $\left\{\tilde{X}_{i}, 0 \leq i \leq L\right\}$, where $L$ is the number of $M$-frames in the sequence, then the number of cells in an $M$-frame can be represented as

$$
\tilde{X}_{i}=\sum_{j=1}^{M} R_{j}^{(i)}
$$

where $R_{j}^{(i)} \in\left\{R_{l}, R_{h}\right\}$ is the instant rate of mini-source $j$ at time slot $i$. Suppose the number of original frames in an $M$ frame is $K$, then equation (1) will be of the following form:

$$
\tilde{Y}_{n}=\sum_{i=0}^{n}\left(\tilde{X}_{i}-K C\right)=\sum_{j=1}^{M} \sum_{i=0}^{n}\left(R_{j}^{(i)}-K C / M\right) .
$$

Obviously, for all $n=0,1, \ldots, L, \tilde{Y}_{n}$ is a random variable. Suppose $\tilde{\mathbf{Y}}_{n}$ is a set that is composed of all the possible values of $\tilde{Y}_{n}$. Define a new random variable $\tilde{Y}$ as follows:

$$
\tilde{Y}=\max \left\{\tilde{Y}_{n}, 0 \leq n \leq L\right\}
$$

Then, the value set of $\tilde{Y}$ is $\tilde{\mathbf{Y}}=\mathrm{U}_{n=0}^{L} \tilde{\mathbf{Y}}_{n}$. Based on the above definitions, our problem becomes: for an arbitrarily small value $\varepsilon$,

1. find a $B_{t}$ such that $P\left\{\tilde{Y}>B_{t}\right\} \leq \varepsilon$; and

2. find a $B_{r 1}$ such that $P\left\{\tilde{Y}<-B_{r 1}\right\} \leq \varepsilon$.

Therefore, the key is to find the probability distribution of $\tilde{Y}$. From the difinition of the D-MMDP model [10] and equation (9), it can be seen that random variable $\tilde{Y}_{n}$ is the sum of $M$ identical and independent random variables. Thus, random variable $\tilde{Y}$ will also be the sum of $M$ identical and independent random variables. Clearly, for a resonably large $M$ (e.g. $M$ is between 10 and 20 in most of the D-MMDP models), we can use a normal distribution to approximate the real distribution of $\tilde{Y}$. Thus, the remaining problem is to find the mean and variance of $\tilde{Y}$.

Let us start with every individual component of equation (7). Suppose $r_{l}=R_{l}-K C / M, r_{h}=R_{h}-K C / M$, and let

$$
Z_{n}=\sum_{i=0}^{n}\left(R_{j}^{(i)}-K C / M\right)
$$

and $\mathbf{Z}_{n}$ is the set of all possible values of $Z_{n}$. Similar to what was done for $\tilde{Y}_{n}$, we define a random variable

$$
Z=\max \left\{Z_{n}, 0 \leq n \leq L\right\},
$$

with values taken from $\mathbf{Z}=\mathrm{U}_{n=0}^{L} \mathbf{Z}_{n}$. Then, $\tilde{Y}$ is the sum of $M Z \mathrm{~s}$.

Since each of the $M$ random variables $Z$ s corresponds to a mini-source in the D-MMDP model, it is easy to obtain from the mini-source model that

$$
\begin{aligned}
& P\left\{Z_{0}=r_{l}\right\}=\beta /(\alpha+\beta), \\
& P\left\{Z_{0}=r_{h}\right\}=\alpha /(\alpha+\beta) .
\end{aligned}
$$

Also, it can be seen that

$$
\begin{aligned}
& P\left\{Z_{n+m}=n r_{l}+m r_{h}\right\}= \\
& \quad P\left\{Z_{0}=r_{l}\right\} A(n-1, m)(1-\alpha)^{n-1} \alpha^{m}+ \\
& \quad+P\left\{Z_{0}=r_{h}\right\} A(n, m-1)(1-\beta)^{m-1} \beta^{n}
\end{aligned}
$$

where $m, n=1,2, \ldots(m+n \leq L)$ and $A(n-1, m)$ is the number of diferent approaches from the initial $Z_{0}=r_{l}$ to $Z_{n+m}=n r_{l}+m r_{h}$ and $A(n, m-1)$ is the number of different approaches from the initial $Z_{0}=r_{h}$ to $Z_{n+m}=n r_{l}+m r_{h}$ and they can be calculated iteratively by the following equations:

$$
\begin{gathered}
A(0,0)=1 \\
A(n, m)=A(m, n)=\sum_{i=0}^{m} A(n-1, i)
\end{gathered}
$$

Up to now, we have obtained the probabilities of all the elements of $Z$. The mean and variance of $Z$ can then be calculated by

$$
\begin{gathered}
\mu_{Z}=\sum_{n+m \leq L} P\left\{Z_{n+m}=n r_{l}+m r_{h}\right\}\left(n r_{l}+m r_{h}\right), \\
\sigma_{Z}^{2}=\sum_{n+m \leq L} P\left\{Z_{n+m}=n r_{l}+m r_{h}\right\}\left(n r_{l}+m r_{h}-\mu_{Z}\right)^{2} .
\end{gathered}
$$

Finally, the mean and variance of $\tilde{Y}$ are respectively

$$
\begin{gathered}
\mu_{\tilde{Y}}=M \mu_{Z}, \\
\sigma_{\tilde{Y}}^{2}=M \sigma_{Z}^{2} .
\end{gathered}
$$

Having the above two parameters, we can then use standard Gaussian approximation formula (see, for example, [5]) to find $B_{t}$ and $B_{r 1}$ such that $P\left\{\tilde{Y}>B_{t}\right\} \leq \varepsilon$ and $P\left\{\tilde{Y}<-B_{r 1}\right\} \leq \varepsilon$ for small $\mathcal{E}$. They are given as

$$
B_{t}=B_{r 1}=\mu_{\tilde{Y}}+\sigma_{\tilde{Y}} \sqrt{-\ln (2 \pi)-2 \ln \varepsilon}
$$

Besides the above analysis, another more straightforward way to solve the problems is to use simulation to find the proper buffer sizes. The following are the simulation results for the real VBR MPEG-2 sequences mentioned in section 2. Figure 1 shows the relationship between the initial playback delay and the required transportation bandwidth. It can be seen that the initial playback delay decreases sharply with the increase in bandwidth. The dashed line marked point corresponds to the mean rate of the sequence. When the transportation rate equals about $8.25 \times 10^{5}$ bps, only about $3 \%$ above the sequence mean rate, the delay becomes almost negligible. This implies that even a small amount of initial playback delay can result in significant savings in bandwidth.

Figure 2 shows the relationship between the required buffer size and the given bandwidth. In this figure, the short dashed line marked point corresponds to the mean rate of the video sequence. It can be seen that the required buffer size almost linearly decreases with the increase in bandwidth when the given bandwidth is less than the mean rate of the video sequence, and the curve reaches the bottom point slightly before reaching the mean video rate. The reason behind is that when the bandwidth is less than the mean video sequence rate, the required buffer size is determined by the number of cells 
accumulated in the initial playback delay period. After the bottom point, when the given bandwidth is larger than the mean video sequence rate, the required buffer size almost linearly increases with the increase in bandwidth and this is because the required buffer size is determined by the number of accumulated cells arriving perior to their scheduled decoding time in these cases.

\section{Controlled CBR Transportation}

The pure CBR transportation scheme discussed in the last section requires a very large buffer. In real applications, it is sometimes difficult or not always possible to satisfy this requirement and a limited buffer size is usually the case. One possible method is to introduce control into the scheme so that the transmission rate can be adjusted according to the buffer condition. More specifically, the controlled scheme is briefly as follows: if the buffer is not full, the transportation is carried out in the given rate $C$. If the buffer is full, the transmission rate is then adjusted to a rate $X=\min \left\{C, X_{n}\right\}$. This control process is not difficult to implement and does not need any feedback information from the receiver. However, the buffer must be large enough to store all the cells arriving in the initial playback delay period. This is one of the issues need to be addressed in this section.

In the controlled CBR scheme, some cells may be delayed due to the rate control and these cells will be out of date when they arrive at the receiver side. Hence, they will be eventually dropped and cause the same effect as the lost cells. This is called rate-control-caused cell loss and is closely related to the given transmission rate and the given buffer size. This relationship is another issue.

Eventhough the control process itself is not complicated, it is still very difficult to find the theoretical solutions to the above issues. Therefore, simulation is used instead. The video sequence used in the simulation is the one discussed in section 2. First of all, let us consider the minimum buffer size required by the scheme. As pointed out before, the minimum buffer size is determined by the initial playback delay,

$$
B_{\text {min }}=D C \text {, }
$$

where $D$ is the initial playback delay and $C$ is the given bandwidth. Figure 3 shows the simulated result of $B_{\min }$ as a function of $C$. In the figure, the dashed line marked point corresponds to the mean rate of the video sequence. It can be seen clearly that the whole curve monotonously decreases with increase in transportation rate $C$ but with different slopes for the $C$ that is less than the mean rate of the video sequence and the $C$ larger than the mean rate of the video sequence.

Figure 4 shows the relationship between the receiver buffer size and the control caused cell loss ratio of delayed-cell dropping at receiver scheme. From the top curve downwards, the $j$ th curve corresponds to the bandwidth equal to $1+(j-1) \%$ of the mean rate of the video sequence. Clearlu, cell loss ratio is unacceptable if the bandwidth equals the mean rate of the video sequence (top curve). If the bandwidth is increased by even a small amount (less than $10 \%$ of the mean rate), an extremely small cell loss ratio can be achieved. Aslo, there is only very little difference between the receiver buffer size required to satisfy a $10^{-2}$ cell loss ratio and a $10^{-10}$ cell loss ratio. This implies that the required receiver buffer size is not very sensitive to the required control caused cell loss ratio when it is large enough to satisfy a certain (say $10^{-6}$ ) cell loss requirement. By comparing all the curves, it can be seen clearly that increasing bandwidth can lead to a great decrease in required receiver buffer size.

\section{Conclusion}

In this paper, we proposed a CBR transportation scheme for VBR MPEG-2 video transportation in ATM networks. It is found that the proposed CBR scheme can save a lot of bandwidth by allowing a certain amount of initial playback delay and a certain receiver buffer. A controlled CBR scheme is also proposed for the applications with limited receiver buffer size. For this controlled CBR scheme, the trade-off between transportation bandwidth and receiver buffer size, cell loss ratio and receiver buffer size are respectively studied. From both network management and bandwidth saving points of view, the porposed scheme is prior to other schemes. However, it can only be used in video on-demand applications. The proposed scheme can obviously be used in other non-real time VBR transportations in ATM networks as well.

\section{References}

[1] H. Chu, D.H.K. Tsang, T. Yang, "Call Admission Control of Teleconferencing VBR Video Traffic in ATM Networks", ICC'95.

[2] A.I. Elwalid, D. Mitra,"Effective Bandwidth of General Markovian Traffic Sources and Admission Control of High Speed Networks", IEEE/ACM Trans. on Networking, Vol.1, June 1993, pp.329-343.

[3] R.J. Gibbens and P.J.Hunt, "Effective Bandwidth for Multi-type UAS Channels", Queueing Systems, Vol.9, 1991, pp.17-28.

[4] M. R. Grasse and J. F. Frater ,"Arnold Traffic Characteristics of MPEG 2 Variable Bit Rate Video", Australian Telecommunication Networks \& Applications Conference, Dec. 1994, pp 473-478.

[5] R. Guerin, H. Ahmadi, M. Naghshineh, "Equivalent Capacity and Its Application to Bandwidth Allocation in High-speed Networks", IEEE JSAC, Vol.9, No.7, Sept., 1991, pp.968-981.

[6] Z. Guo, J. Ni and T. Yang, "Traffic Smoothing and Bandwidth Allocation for VBR MPEG-2 Video Connections in ATM Networks", IEEE ICCCN'95, pp554-561.

[7] B. Jabbari, F. Yegenoglu, Y. Kuo, S. Zafar and Y. Zhang "Statistical Characterization and Block-Based Modeling of Motion-Adaptive Coded Video" IEEE Transactions on Circuits and Systems for Video Technology, Vol.3, No.3, June 1993, p199-207.

[8] G. Kesidis, J. Walrand, C.S. Chang, "Effective Bandwidth for Multiclass Markov Fluids and Other ATM Sources", IEEE/ACM Trans. on Networking, Vol.1, August, 1993, pp.424-428.

[9] M. Krunz, R. Sass, and H. Hughes, "Statistical Characteristics and Multiplexing of MPEG-coded Video Streams", expected in the INFOCOM 1995. 
[10] J. Ni and T. Yang, "Source Modelling, Queueing Analysis and Bandwidth Allocation of VBR MPEG-2 Video Traffic in ATM Networks", IEEE ATM Workshop, Washington D C, Oct. 1995.

[11] P. Pancha and M. EI Zarki, "MPEG Coding For Variable Bit Rate Video Transmission", IEEE Communications Magazine, May 1994, pp54-66.

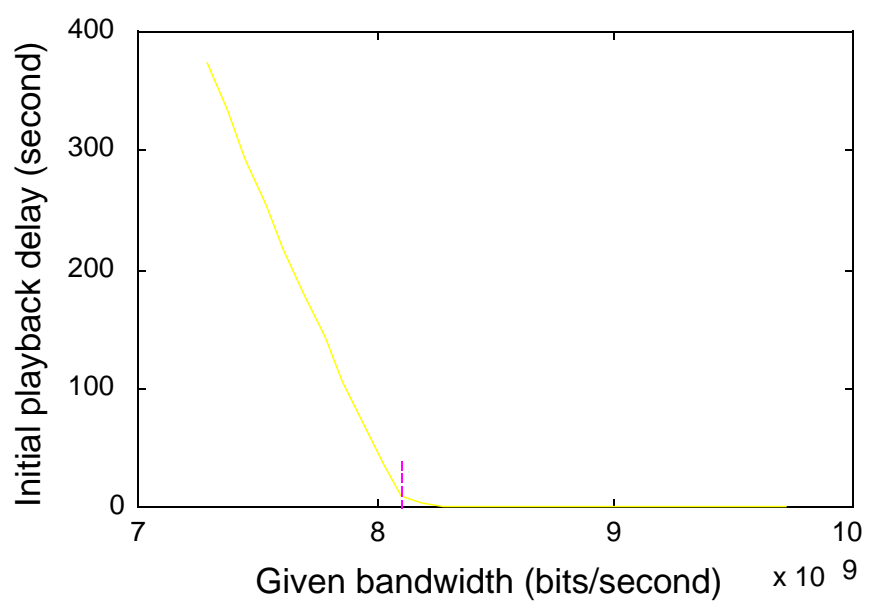

Figure 1. Initial playback delay vs. bandwidth

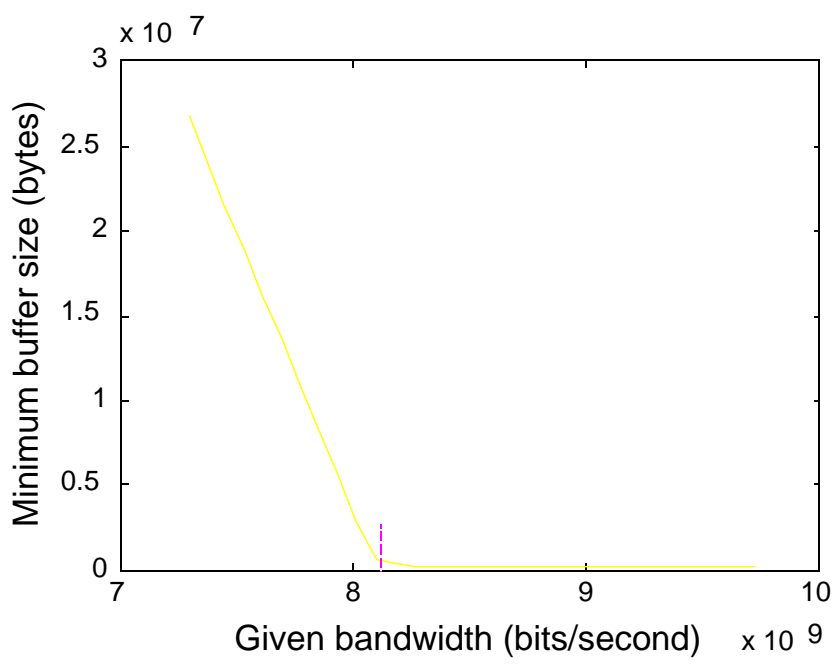

Figure 3. Minimum Receiver buffer size vs. bandwidth
[12] D. Reininger and D. Raychaudhuri, "Bit-Rate Characteristics of a VBR MPEG Video Encoder for ATM Networks", ICC 93, pp.517-521.

[13] T. Yang, D. H.K. Tsang, and S.Y. Li,"Cell Scheduling and Bandwidth Allocation for a class of VBR Video Connections", IEEE Workshop Visual Signal Processing and Commun., Sept, 1994, pp95-101.

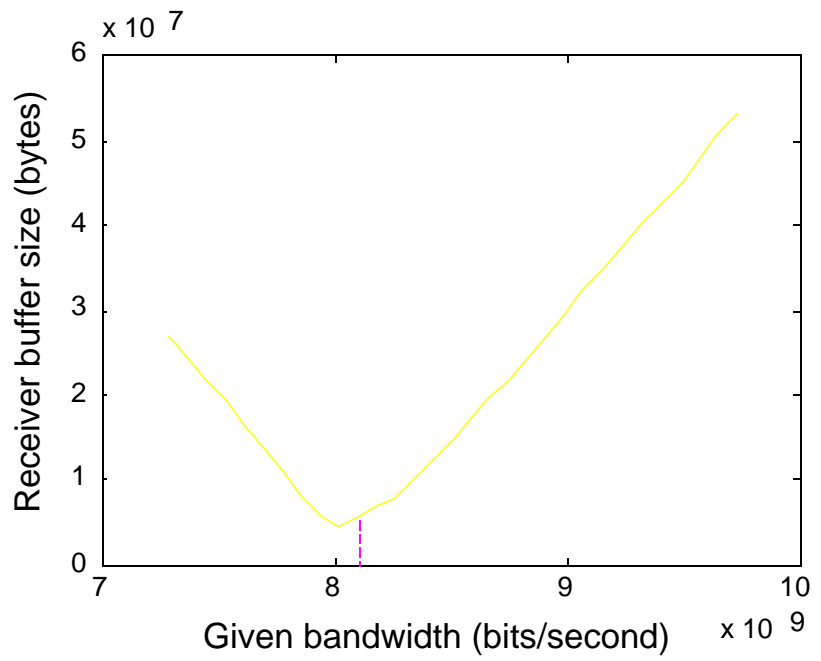

Figure 2. Receiver buffer size vs. bandwidth

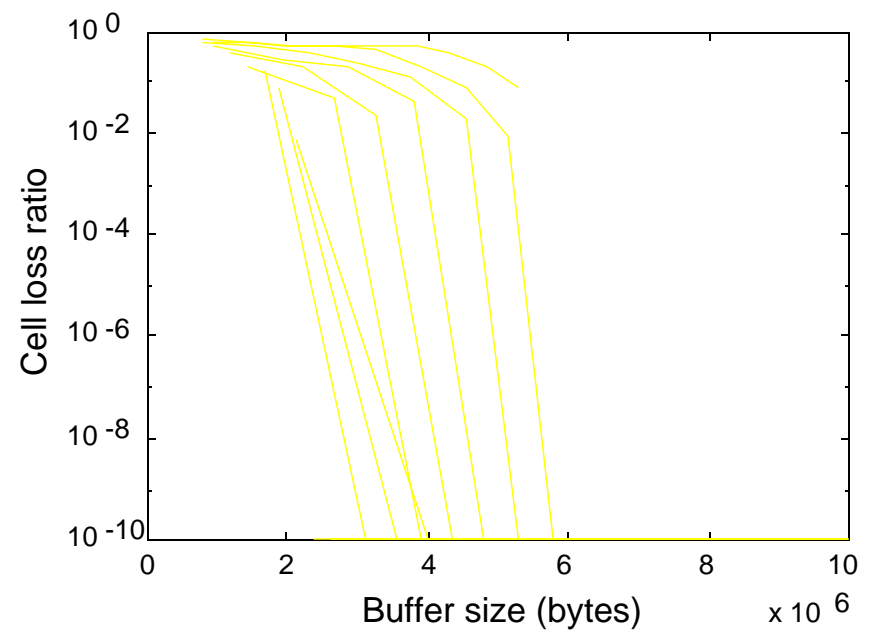

Figure 4. Cell loss ratio vs. receiver buffer size 